\title{
COMPARAÇÃO DE TÉCNICAS ORTODÔNTICAS E UMA SUGESTÃO DE TRATAMENTO PARA O PACIENTE ATLETA ${ }^{1}$
}

\author{
COMPARISON OF ORTHODONTIC TECHNIQUES AND A \\ TREATMENT SUGGESTION FOR THE ATHLETE PATIENT
}

\section{Ary Ferreira Nunes ${ }^{2}$, Carlos Lane Fogaça ${ }^{3}$, Rodrigo Stanislawczuk Grande ${ }^{4}$, Eduardo Guaragna Kayser ${ }^{5}$ e Barbara Capitanio de Souza ${ }^{6}$}

\section{RESUMO}

A ortodontia tradicional foi desenvolvida através da colagem dos braquetes na face vestibular dos elementos dentários e, durante décadas, predominou como a técnica de eleição. Quando pensamos nas particularidades individuais dos pacientes, encontramos no atleta um perfil que requer importantes considerações sobre o tratamento ortodôntico. Estes indivíduos estão mais suscetíveis aos eventos traumáticos na região orofacial e possuem uma rotina de treinamento e competições que pode contribuir para o aumento da vulnerabilidade às doenças bucais. Pensando em avaliar melhor as possibilidades de tratamento ortodôntico para o paciente atleta, o objetivo deste estudo foi realizar uma revisão da literatura sobre as vantagens e desvantagens da ortodontia fixa com braquetes e comparar com a técnica Bracketless Orthodontic Treatment. Para isto, foi realiza uma revisão de literatura incluindo artigos indexados na base de dados PubMed e selecionados por busca manual. A pesquisa utilizou os termos em inglês: ortodontia fixa, aparelho ortodôntico, braquetes ortodônticos, traumatismo oral, lesões bucais, higiene oral, cáries, gengivite e atleta. Ao final das análises, foram selecionados 15 estudos. Os resultados mostraram as vantagens e as desvantagens das técnicas tradicionais em comparação com a técnica Bracketless Orthodontic Treatment. Os braquetes ortodônticos são responsáveis pelo aumento do risco de doenças bucais como a cárie e a gengivite e podem favorecer a gravidade de traumas orofaciais. A técnica Bracketless Orthodontic Treatment pode ser uma opção de tratamento ortodôntico para o esportista, pois além de reduzir os riscos de injúrias às mucosas orais, permite uma boa higienização dos dentes.

Palavras-chave: Ortodontia, Traumatismo dentário, Protetores bucais, Educação física e treinamento.

\section{ABSTRACT}

Traditional orthodontics was developed by bonding brackets to the buccal surface of dental elements and, for decades, it predominated as the technique of choice. When we think about the individual characteristics of patients, we find in the athlete a profile that requires important considerations about orthodontic treatment. These individuals are more susceptible to traumatic events in the orofacial region and have a training and competition routine that can contribute to the increased vulnerability to oral diseases. Aiming to better evaluate the possibilities of orthodontic treatment for athlete patients, the aim of this study was to review

\footnotetext{
${ }^{1}$ Trabalho de Conclusão de Curso.

${ }^{2}$ Especializando do Curso de Odontologia do Esporte - Associação Brasileira de Odontologia - ABORS. E-mail: aryfnunes@gmail.com

${ }^{3}$ Especializando do Curso de Odontologia do Esporte - Associação Brasileira de Odontologia - ABORS. E-mail: drfogaca@msn.com

${ }^{4}$ Especializando do Curso de Odontologia do Esporte - Associação Brasileira de Odontologia - ABORS. E-mail: rodrigozuk1@hotmail.com

${ }^{5}$ Coordenador. Docente do Curso de Especialização em Odontologia do Esporte - Associação Brasileira de Odontologia ABORS. E-mail: eduardogkayser@hotmail.com

${ }^{6}$ Coordenadora. Docente do Curso de Especialização em Odontologia do Esporte - Associação Brasileira de Odontologia ABORS. E-mail: barbara.capitanio@gmail.com
} 
the literature on the advantages and disadvantages of fixed orthodontics with brackets and to compare them with the Bracketless Orthodontic Treatment technique. For this, a literature review was carried out including articles indexed in the PubMed database and selected by manual search. The research used the terms in English: fixed orthodontics, orthodontic appliance, orthodontic brackets, oral trauma, oral lesions, oral hygiene, caries, gingivitis and athlete. At the end of the analysis, 15 studies were selected. The results showed the advantages and disadvantages of traditional techniques compared to the Bracketless Orthodontic Treatment technique. Orthodontic brackets are responsible for the increased risk of oral diseases such as caries and gingivitis and can favor the severity of orofacial trauma. The Bracketless Orthodontic Treatment technique can be an orthodontic treatment option for the sportsman, because in addition to reducing the risk of injury to the oral mucous membranes, it allows good teeth hygiene.

Keywords: Orthodontics, Tooth injuries, Mouthguard, Physical education and training.

\section{INTRODUÇÃO}

O tratamento ortodôntico convencional foi desenvolvido através da colagem dos braquetes, que são acessórios ortodônticos, na face vestibular dos elementos dentários e, durante décadas, predominou como a técnica de eleição dos ortodontistas. Com o passar dos anos, houve um aumento do número de pacientes que procuram o tratamento ortodôntico, assim como da busca por soluções mais estéticas. Estes fatores impulsionaram o desenvolvimento de pesquisas e novas técnicas, com diferentes abordagens, como os alinhadores estéticos e aparelhos com braquetes fixados na face lingual dos dentes (LONG et al., 2013; MISTAKIDIS et al., 2016). De fato, o aperfeiçoamento das técnicas ortodônticas apresentaram vantagens interessantes para as demandas apresentadas naquele momento, contudo, quando pensamos ainda nas questões de escolha dos pacientes, a ortodontia fixa permanece como eleição, possivelmente pela consideração dos custos (PAPAGEORGIOU et al., 2016).

Ao analisarmos as particularidades dos pacientes, encontramos no atleta um perfil que requer importantes considerações sobre este tipo de tratamento. Estes indivíduos estão mais suscetíveis aos eventos traumáticos na região orofacial (QUDEIMAT et al., 2019), possuem uma rotina de treinamento e competições que podem contribuir para o aumento da vulnerabilidade às doenças bucais (TIBANA et al., 2019), frequentemente utilizam formas de suplementação alimentar com valores elevados de carboidratos e de sacarose (TOUGER-DECKER; VAN LOVEREN, 2003) e consomem bebidas do tipo repositores que apresentam alto teor de acidez (VENABLES et al., 2005). Além disso, a ocorrência de eventuais solturas de braquetes durante viagens pode, além de interferir no momento do tratamento, causar desconfortos e produzir machucaduras e dores indesejáveis (SHEN et al., 2016). Todos estes elementos caracterizam o atleta como um paciente que apresenta fatores de riscos diferenciados para os problemas odontológicos, o que nos faz reavaliar a aplicação da ortodontia fixa convencional, neste caso. Além disso, a literatura vigente ainda é bastante escassa, quando pesquisamos sobre este tema, o que também limita, de certa forma, a aplicação clínica. 
Como a ortodontia fixa vestibular ou técnica labial ocasionava uma maior gravidade durante as ocorrências de traumas orais, em um primeiro momento, a ortodontia fixa lingual se tornou a técnica de escolha, principalmente para o uso em atletas. Entretanto, o aumento da proteção foi observado apenas sobre os tecidos moles labiais. Lesões associadas à língua e ao assoalho bucal ainda permaneceram presentes, além de outras queixas como a maior dificuldade de higienização, maior dificuldade na adaptação para comunicação e alimentação (WU et al., 2008; SHALISH et al., 2012; LONG et al., 2013) e a confecção de protetores bucais muito volumosos na região lingual, fazendo com que muitos atletas não aderissem muito ao seu uso (PARKER et al, 2017). Conhecendo os fatores de risco comportamentais e o estilo de rotina do esportista, a ortodontia fixa lingual acaba apresentando consideráveis pontos de desvantagens como opção de tratamento, para esta população.

Os aparelhos do tipo alinhadores estéticos poderiam ser uma boa alternativa para o paciente atleta; porém, esta modalidade de tratamento exige certa disciplina de uso e seus custos podem estar fora das possibilidades da maior parte desta população. Como um contraponto alternativo de tratamento, temos a técnica Bracketless Orthodontic Treatment (BOT), que preconiza uma ortodontia fixa sem o uso de braquetes. Esta alternativa de tratamento acompanha vantagens particulares e muito interessantes, quando analisamos as necessidades do atleta, como não interferir na fonética, possibilitar uma higienização mais precisa, não causar danos aos tecidos orais, viabilizar a confecção de protetores bucais menos volumosos, além de possuir um custo mais acessível (MUSILLI, 2008; MUSILLI et al., 2012). Pensando em avaliar melhor as possibilidades de tratamento ortodôntico para o paciente atleta, o objetivo deste estudo foi realizar uma revisão da literatura sobre as vantagens e desvantagens da ortodontia fixa com braquetes e comparar com a técnica BOT.

\section{METODOLOGIA}

Este é um estudo descritivo analítico, que foi realizado através de revisão de literatura. A pesquisa foi desenvolvida entre os meses de janeiro e fevereiro de 2020 e incluiu os artigos indexados na base de dados PubMed e selecionados por busca manual. Não houve restrição para o ano de publicação. A busca de artigos foi realizada utilizando as seguintes combinações de termos em inglês: ortodontia fixa (fixed orthodontics), aparelho ortodôntico (orthodontic appliance), braquetes ortodônticos (orthodontic brackets), traumatismo oral (oral trauma), lesões bucais (oral injuries), higiene ora (oral hygiene), cáries (dental caries), gengivite (gengivitis) e atleta (athlete) (Tabela 1). Os artigos selecionados incluíram população de atletas e não atletas, uma vez que o objetivo do trabalho está direcionado especialmente às comparações entre as técnicas ortodônticas. A população alvo do estudo são indivíduos que fazem uso de aparelhos ortodônticos com braquetes, não sendo necessariamente exposta ao exercício físico, pois a literatura é escassa para estes indivíduos. Após a análise do 
título e do resumo disponível, foram excluídos os artigos que apresentavam casos clínicos, que não tratavam do tema proposto, que não estavam publicados em inglês, que consideravam outros tipos de aparelhos ortodônticos móveis ou que não disponibilizavam o texto completo. Ao final da análise, foram selecionados 15 estudos para comporem a revisão final (Figura 1). Os estudos selecionados foram apresentados salientando as suas principais características e informações (Quadro 1).

Tabela 1 - Combinação dos termos para a busca inicial dos artigos indexados.

\begin{tabular}{|lc}
\hline \multirow{2}{*}{ Combinação de Termos } & Quantidade de artigos \\
\cline { 2 - 2 } & PubMed e busca manual \\
\hline Fixed orthodontics and oral trauma & 16 \\
\hline Fixed orthodontics and oral injuries & 11 \\
\hline Fixed orthodontics and athlete & 3 \\
\hline Orthodontic brackets and oral injuries & 177 \\
\hline Orthodontic brackets and oral trauma & 199 \\
\hline Orthodontic brackets and athlete & 0 \\
\hline Orthodontic appliance and oral injuries & 118 \\
\hline Orthodontic appliance and oral trauma & 44 \\
\hline Orthodontic appliance and athlete & 9 \\
\hline Fixed orthodontics and oral hygiene & 388 \\
\hline Fixed orthodontics and dental caries & 58 \\
\hline Fixed orthodontics and gengivitis & 22 \\
\hline Total & $\mathbf{1 . 0 4 5}$ \\
\hline
\end{tabular}

Fonte: construção do autor.

Figura 1 - Sistemática da seleção dos estudos.

Busca inicial na base de dados, com a combinação de descritores:1.045 estudos.

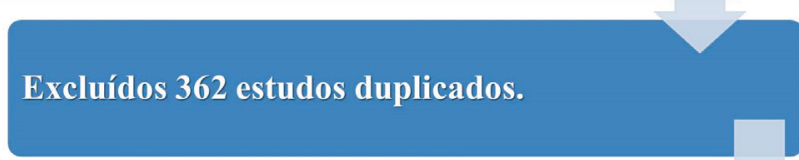

Excluídos 634 estudos, após avaliação dos títulos e dos resumos disponíveis.

Excluídos 29 estudos, após leitura e análise do texto completo.

Incluídos 15 estudos na revisão final.

Fonte: Construção do autor. 
Quadro 1 - Resumo das características dos artigos utilizados para a revisão.

\begin{tabular}{|c|c|c|c|c|c|c|}
\hline Autor & Título & Objetivo & $\begin{array}{l}\text { Delinea- } \\
\text { mento }\end{array}$ & Amostra & Principais Resultados & Conclusão \\
\hline $\begin{array}{l}\text { KVITTEN } \\
\text { et al., } 1998\end{array}$ & $\begin{array}{l}\text { Incidence } \\
\text { of Orofacial } \\
\text { Injuries in High } \\
\text { School Sports }\end{array}$ & $\begin{array}{l}\text { Determinar a incidên- } \\
\text { cia de lesões orofaciais } \\
\text { em esportes do ensino } \\
\text { médio, comparar taxas } \\
\text { de lesões e examinar a } \\
\text { relação entre o uso de } \\
\text { aparelhos ortodônticos } \\
\text { fixos e a ocorrência de } \\
\text { lesões orofaciais. }\end{array}$ & $\begin{array}{l}\text { Estudo } \\
\text { descritivo } \\
\text { epidemioló- } \\
\text { gico }\end{array}$ & $\begin{array}{l}318 \text { atletas } \\
\text { juvenis }\end{array}$ & $\begin{array}{l}\text { Os aparelhos ortodônticos } \\
\text { fixos apresentaram maior } \\
\text { risco de sofrer uma lesão em } \\
\text { todos os esportes. Metade } \\
\text { dos atletas sabem da impor- } \\
\text { tância do uso de protetores } \\
\text { bucais, mas apenas } 6 \% \text { dos } \\
\text { atletas relatam o seu uso. }\end{array}$ & $\begin{array}{l}\text { Observou-se uma } \\
\text { alta taxa de lesões } \\
\text { orofaciais, relatadas } \\
\text { pelos atletas e o uso do } \\
\text { aparelho fixo ortodôn- } \\
\text { tico pode aumentar a } \\
\text { gravidade das lesões. }\end{array}$ \\
\hline $\begin{array}{c}\text { BARICEVIC } \\
\text { et al., } 2011\end{array}$ & $\begin{array}{l}\text { Oral mucosal } \\
\text { lesions during } \\
\text { orthodontic } \\
\text { treatment }\end{array}$ & $\begin{array}{l}\text { Examinar a frequência } \\
\text { das lesões em mucosas } \\
\text { orais de usuários de } \\
\text { aparelhos ortodônticos } \\
\text { e comparar com crian- } \\
\text { ças com má oclusão. }\end{array}$ & $\begin{array}{l}\text { Estudo } \\
\text { Caso-con- } \\
\text { trole }\end{array}$ & $\begin{array}{l}111 \\
\text { pacientes, } \\
\text { sendo } 60 \\
\text { casos e } 51 \\
\text { controles }\end{array}$ & $\begin{array}{l}\text { As lesões mucosas, erosões } \\
\text { e ulcerações foram mais } \\
\text { frequentes e presentes } \\
\text { nos usuários de aparelhos } \\
\text { ortodônticos fixos, prin- } \\
\text { cipalmente relacionados a } \\
\text { traumas sobre o aparelho. }\end{array}$ & $\begin{array}{l}\text { O tratamento ortodôn- } \\
\text { tico leva a um } \\
\text { maior risco de ocorrer } \\
\text { lesões nas mucosas e } \\
\text { requer maior cuidado } \\
\text { com a higiene bucal. }\end{array}$ \\
\hline $\begin{array}{l}\text { AHMED; } \\
\text { SAIF-UL- } \\
\text {-HAQUE; } \\
\text { NAZIR, } 2011\end{array}$ & $\begin{array}{l}\text { Carious lesions } \\
\text { in patients } \\
\text { undergoing } \\
\text { orthodontic } \\
\text { treatment }\end{array}$ & $\begin{array}{l}\text { Registrar as lesões } \\
\text { cariosas recém-desen- } \\
\text { volvidas em pacientes } \\
\text { que recebem tratamen- } \\
\text { to ortodôntico fixo. }\end{array}$ & $\begin{array}{l}\text { Estudo } \\
\text { transversal }\end{array}$ & $\begin{array}{l}90 \\
\text { pacientes } \\
\text { ortodônti- } \\
\text { cos }\end{array}$ & $\begin{array}{l}\text { A frequência de cárie } \\
\text { aumentou com o aumento } \\
\text { do período de tratamento } \\
\text { ortodôntico. Pacientes após } \\
\text { o tratamento de } 12 \text { meses } \\
\text { teve mais lesões de cárie } \\
\text { do que aos } 6 \text { meses. }\end{array}$ & $\begin{array}{l}\text { O paciente ortodôntico } \\
\text { precisa ser mais mo- } \\
\text { tivado em relação aos } \\
\text { cuidados com a saúde } \\
\text { bucal. }\end{array}$ \\
\hline $\begin{array}{c}\text { CONTI } \\
\text { et al., } 2012\end{array}$ & $\begin{array}{l}\text { Aesthetic } \\
\text { restoration } \\
\text { of upper lip } \\
\text { after removal } \\
\text { of post-trauma } \\
\text { foreign body } \\
\text { (orthodontic } \\
\text { bracket) }\end{array}$ & $\begin{array}{l}\text { Descrever um caso de } \\
\text { soltura de um braquete } \\
\text { e inclusão no lábio } \\
\text { superior, após evento } \\
\text { traumático. }\end{array}$ & $\begin{array}{l}\text { Relato de } \\
\text { Caso }\end{array}$ & $\begin{array}{l}1 \text { mulher } \\
\text { de } 18 \text { anos }\end{array}$ & $\begin{array}{l}\text { Em caso de trauma orofa- } \\
\text { cial em paciente que utiliza } \\
\text { aparelho fixo, é importante } \\
\text { a precisão do diagnóstico e } \\
\text { terapia de tecidos duros e } \\
\text { moles, para que a recons- } \\
\text { trução estética possa ser al- } \\
\text { cançada e os tecidos moles } \\
\text { possam cicatrizar em um } \\
\text { curto período de tempo. }\end{array}$ & $\begin{array}{l}\text { Após traumas orofa- } \\
\text { ciais, é muito im- } \\
\text { portante a adequada } \\
\text { inspeção dos tecidos, } \\
\text { em especial nos casos } \\
\text { envolvendo aparelhos } \\
\text { com braquetes. }\end{array}$ \\
\hline $\begin{array}{l}\text { MCCARTHY; } \\
\text { COBB, } 2012\end{array}$ & $\begin{array}{l}\text { Orthodontic } \\
\text { parotitis: a } \\
\text { rare complica- } \\
\text { tion from an } \\
\text { orthodontic } \\
\text { appliance }\end{array}$ & $\begin{array}{l}\text { Relatar um caso de } \\
\text { estase salivar secun- } \\
\text { dária à inflamação da } \\
\text { mucosa jugal causada } \\
\text { por trauma do braque- } \\
\text { te ortodôntico. }\end{array}$ & $\begin{array}{l}\text { Relato de } \\
\text { Caso }\end{array}$ & $\begin{array}{l}1 \text { mulher } \\
\text { de } 14 \text { anos }\end{array}$ & $\begin{array}{l}\text { As peças dos aparelhos } \\
\text { ortodônticos fixos podem } \\
\text { causar lesões na mucosa } \\
\text { oral e comprometer a } \\
\text { funcionalidade de órgãos } \\
\text { adjacentes. }\end{array}$ & $\begin{array}{l}\text { Deve-se ter cuidado } \\
\text { como uso de apare- } \\
\text { lhos fixos, para evitar } \\
\text { lesões nos tecidos. É } \\
\text { importante conhecer os } \\
\text { potenciais risco do tra- } \\
\text { tamento e saber realizar } \\
\text { o manejo das situações } \\
\text { de urgências. }\end{array}$ \\
\hline
\end{tabular}




\begin{tabular}{|c|c|c|c|c|c|c|}
\hline $\begin{array}{l}\text { TANNER } \\
\text { et al., } 2012\end{array}$ & $\begin{array}{l}\text { White-spot } \\
\text { Lesions and } \\
\text { Gingivitis } \\
\text { Microbiotas in } \\
\text { Orthodontic } \\
\text { Patients }\end{array}$ & $\begin{array}{l}\text { Avaliar a microbiota } \\
\text { da lesão de mancha } \\
\text { branca em pacientes } \\
\text { ortodônticos como } \\
\text { modelo de desenvolvi- } \\
\text { mento inicial de cárie } \\
\text { em crianças e a relação } \\
\text { entre a microbiota das } \\
\text { lesões e da gengivite. }\end{array}$ & $\begin{array}{l}\text { Estudo } \\
\text { Caso-con- } \\
\text { trole }\end{array}$ & $\begin{array}{l}60 \\
\text { pacientes } \\
\text { entre } 10 \\
\text { e } 19 \text { anos, } \\
\text { sendo } 30 \\
\text { casos e } 30 \\
\text { controles }\end{array}$ & $\begin{array}{l}\text { Observou-se uma diver- } \\
\text { sidade de microbiologia } \\
\text { da placa dentária e a sua } \\
\text { relação de S. mutans e com } \\
\text { ortodontia fixa. Houve tam- } \\
\text { bém uma maior presença } \\
\text { de patógenos periodontais } \\
\text { com o uso de aparelho. }\end{array}$ & $\begin{array}{l}\text { O uso de aparelho } \\
\text { fixo ortodôntico pode } \\
\text { favorecer o aumento da } \\
\text { microbiota oral. }\end{array}$ \\
\hline $\begin{array}{l}\text { ZURFLUH; } \\
\text { WAES; } \\
\text { FILIPPI, } 2013\end{array}$ & $\begin{array}{l}\text { The Influence } \\
\text { of Fixed } \\
\text { Orthodontic } \\
\text { Appliances on } \\
\text { Halitosis }\end{array}$ & $\begin{array}{l}\text { Investigar a influência } \\
\text { de aparelhos } \\
\text { ortodônticos fixos na } \\
\text { ocorrência de halitose. }\end{array}$ & $\begin{array}{l}\text { Estudo } \\
\text { transversal }\end{array}$ & $\begin{array}{l}55 \\
\text { pacientes } \\
\text { ortodônti- } \\
\cos \end{array}$ & $\begin{array}{l}\text { Na presença do aparelho } \\
\text { fixo com braquetes, houve } \\
\text { um aumento significati- } \\
\text { vo de placa bacteriana e } \\
\text { saburra lingual, potenciali- } \\
\text { zando os sinais de halitose. }\end{array}$ & $\begin{array}{l}\text { A halitose pode ser um } \\
\text { importante indicador } \\
\text { de saúde durante o } \\
\text { tratamento ortodôntico } \\
\text { e pode servir como } \\
\text { fator motivador para o } \\
\text { paciente. }\end{array}$ \\
\hline $\begin{array}{c}\text { MÁRTHA; } \\
\text { MEZEI; } \\
\text { JÁNOSI, } 2013\end{array}$ & $\begin{array}{l}\text { A histological } \\
\text { analysis of } \\
\text { gingival } \\
\text { condition } \\
\text { associated with } \\
\text { orthodontic } \\
\text { treatment }\end{array}$ & $\begin{array}{l}\text { Analisar a reação } \\
\text { gengival em aparelhos } \\
\text { ortodônticos fixos. }\end{array}$ & $\begin{array}{l}\text { Estudo } \\
\text { transversal }\end{array}$ & $\begin{array}{l}11 \\
\text { pacientes }\end{array}$ & $\begin{array}{l}\text { As observações histológicas } \\
\text { demonstraram e confirma- } \\
\text { ram a presença de gengi- } \\
\text { vite durante o tratamento } \\
\text { ortodôntico com a presença } \\
\text { de alterações inflamatórias } \\
\text { crônicas hiperplásicas de } \\
\text { gravidade leve a moderada. }\end{array}$ & $\begin{array}{l}\text { O tratamento ortodôn- } \\
\text { tico fixo pode causar } \\
\text { alterações histológicas } \\
\text { nos tecidos periodontais, } \\
\text { podendo haver maior } \\
\text { gravidade como prolon- } \\
\text { gamento da terapia. }\end{array}$ \\
\hline $\begin{array}{l}\text { ZANATTA } \\
\text { et al., } 2014\end{array}$ & $\begin{array}{l}\text { Association be- } \\
\text { tween gingivitis } \\
\text { and anterior gin- } \\
\text { gival enlarge- } \\
\text { ment in subjects } \\
\text { undergoing } \\
\text { fixed orthodon- } \\
\text { tic treatment }\end{array}$ & $\begin{array}{l}\text { Investigar a associa- } \\
\text { ção entre aumento } \\
\text { gengival, condições } \\
\text { periodontais e carac- } \\
\text { terísticas sociodemo- } \\
\text { gráficas em indivíduos } \\
\text { submetidos a trata- } \\
\text { mento ortodôntico fixo. }\end{array}$ & $\begin{array}{l}\text { Estudo } \\
\text { transversal }\end{array}$ & $\begin{array}{l}330 \\
\text { pacientes } \\
\text { ortodônti- } \\
\cos \end{array}$ & $\begin{array}{l}\text { O excesso de resina ao } \\
\text { redor dos braquetes e a } \\
\text { dificuldade de higieniza- } \\
\text { ção foram associados ao } \\
\text { aumento gengival. }\end{array}$ & $\begin{array}{l}\text { Aparelhos ortodônticos } \\
\text { fixo com braquetes } \\
\text { podem ser um fator de } \\
\text { risco adicional para as } \\
\text { doenças gengivais. }\end{array}$ \\
\hline KHALAF, 2014 & $\begin{array}{l}\text { Factors } \\
\text { Affecting the } \\
\text { Formation, } \\
\text { Severity and } \\
\text { Location of } \\
\text { White Spot } \\
\text { Lesions during } \\
\text { Orthodontic } \\
\text { Treatment } \\
\text { with Fixed } \\
\text { Appliances }\end{array}$ & $\begin{array}{l}\text { Investigar fatores que } \\
\text { afetam a formação, } \\
\text { gravidade e locali- } \\
\text { zação das lesões da } \\
\text { mancha branca em pa- } \\
\text { cientes que completam } \\
\text { terapia com aparelho } \\
\text { fixo. }\end{array}$ & $\begin{array}{l}\text { Estudo } \\
\text { transversal }\end{array}$ & $\begin{array}{l}45 \\
\text { pacientes } \\
\text { ortodônti- } \\
\cos \end{array}$ & $\begin{array}{l}\text { Os pacientes apresentaram } \\
\text { uma prevalência importan- } \\
\text { te de lesões de cáries, sen- } \\
\text { do que esta foi associada à } \\
\text { dificuldade de higienização } \\
\text { do aparelho, ao tempo de } \\
\text { tratamento e à alimentação. }\end{array}$ & $\begin{array}{l}\text { A dificuldade de hi- } \\
\text { giene oral na presença } \\
\text { do aparelho fixo é um } \\
\text { importante fator para } \\
\text { considerar a escolha da } \\
\text { técnica de tratamento } \\
\text { ortodôntico. }\end{array}$ \\
\hline $\begin{array}{c}\text { WILMOTT; } \\
\text { IKEAGWUANI; } \\
\text { MCLEOD, } 2016\end{array}$ & $\begin{array}{l}\text { An orthodontic } \\
\text { bracket embed- } \\
\text { ded in the me- } \\
\text { dial pterygoid } \\
\text { surface: a case } \\
\text { report }\end{array}$ & $\begin{array}{l}\text { Apresentar um caso } \\
\text { em que houve um } \\
\text { desprendimento de } \\
\text { uma peça ortodôntica } \\
\text { com risco para a saúde } \\
\text { do paciente. }\end{array}$ & $\begin{array}{l}\text { Relato de } \\
\text { Caso }\end{array}$ & $\begin{array}{l}1 \text { mulher } \\
\text { de } 19 \text { anos }\end{array}$ & $\begin{array}{l}\text { Materiais odontológicos } \\
\text { ou peças de aparelhos } \\
\text { ortodônticos podem se } \\
\text { desprender e causarem } \\
\text { machucaduras e potenciais } \\
\text { danos ao paciente. }\end{array}$ & $\begin{array}{l}\text { Existe um risco } \\
\text { potencial de braquetes } \\
\text { ortodônticos serem } \\
\text { deglutidos e aspirados } \\
\text { ou, ainda, incorporado } \\
\text { aos tecidos moles. }\end{array}$ \\
\hline
\end{tabular}




\begin{tabular}{|c|c|c|c|c|c|c|}
\hline $\begin{array}{l}\text { SÖKÜCÜ } \\
\text { et al., } 2016\end{array}$ & $\begin{array}{l}\text { The effect } \\
\text { of fixed } \\
\text { appliances } \\
\text { on oral malodor } \\
\text { from beginning } \\
\text { of treatment } \\
\text { till } 1 \text { year }\end{array}$ & $\begin{array}{l}\text { Investigar o aumento } \\
\text { da halitose durante o } \\
\text { uso de aparelhos fixos } \\
\text { ortodônticos. }\end{array}$ & $\begin{array}{l}\text { Estudo } \\
\text { transversal }\end{array}$ & $\begin{array}{l}13 \\
\text { pacientes } \\
\text { ortodônti- } \\
\cos \end{array}$ & $\begin{array}{l}\text { A halitose oral foi significa- } \\
\text { tivamente mais expressiva } \\
\text { entre os pacientes ortodôn- } \\
\text { ticos, em especial a partir } \\
\text { de } 7 \text { meses de tratamento. }\end{array}$ & $\begin{array}{l}\text { O uso dos aparelhos } \\
\text { ortodônticos fixos } \\
\text { aumentam a halitose. }\end{array}$ \\
\hline $\begin{array}{l}\text { SALMERÓN- } \\
\text {-VALDÉS et al., } \\
2016\end{array}$ & $\begin{array}{l}\text { Tooth demi- } \\
\text { neralization } \\
\text { and associated } \\
\text { factors in } \\
\text { patients on } \\
\text { fixed } \\
\text { orthodontic } \\
\text { treatment }\end{array}$ & $\begin{array}{l}\text { Determinar o nível } \\
\text { de desmineralização } \\
\text { dentária em pacientes } \\
\text { durante tratamento } \\
\text { ortodôntico e sua asso- } \\
\text { ciação com pH salivar } \\
\text { e fatores higiênicos e } \\
\text { alimentares. }\end{array}$ & $\begin{array}{l}\text { Estudo } \\
\text { transversal }\end{array}$ & $\begin{array}{l}108 \\
\text { pacientes } \\
\text { em três } \\
\text { diferentes } \\
\text { estágios } \\
\text { de trata- } \\
\text { mento }\end{array}$ & $\begin{array}{l}\text { O tempo de tratamento } \\
\text { ortodôntico com aparelhos } \\
\text { fixos com braquetes acima } \\
\text { de dois anos apresenta } \\
\text { um risco potencial para o } \\
\text { aumento da prevalência e } \\
\text { da gravidade de cáries. }\end{array}$ & $\begin{array}{l}\text { Os padrões de desmi- } \\
\text { neralização encontra- } \\
\text { dos foram os mesmos, } \\
\text { mesmo quando a dieta } \\
\text { e a higiene diferiram, } \\
\text { indicando que o pró- } \\
\text { prio aparelho pode ser } \\
\text { um fator causal isolado. }\end{array}$ \\
\hline $\begin{array}{l}\text { ELTAYEB } \\
\text { et al., } 2017\end{array}$ & $\begin{array}{l}\text { Distribution } \\
\text { of white spot } \\
\text { lesions among } \\
\text { orthodontic pa- } \\
\text { tients attending } \\
\text { teaching } \\
\text { institutes in } \\
\text { Khartoum }\end{array}$ & $\begin{array}{l}\text { Avaliar a prevalência } \\
\text { e padrão de distri- } \\
\text { buição das lesões da } \\
\text { mancha branca entre } \\
\text { pacientes ortodônticos. }\end{array}$ & $\begin{array}{l}\text { Estudo } \\
\text { transversal }\end{array}$ & $\begin{array}{l}158 \\
\text { pacientes } \\
\text { ortodônti- } \\
\cos \end{array}$ & $\begin{array}{l}\text { A prevalência de cárie } \\
\text { identificada foi alta, } \\
\text { confirmando ser um } \\
\text { problema durante o } \\
\text { tratamento ortodôntico. }\end{array}$ & $\begin{array}{l}\text { Pacientes em tratamen- } \\
\text { to ortodôntico em uso } \\
\text { de aparelhos fixos com } \\
\text { braquetes necessitam } \\
\text { de programas de pre- } \\
\text { venção mais rigorosos } \\
\text { e práticas de higiene } \\
\text { bucal frequentes } \\
\text { durante o tratamento } \\
\text { ortodôntico. }\end{array}$ \\
\hline $\begin{array}{c}\text { MEI } \\
\text { et al., } 2017\end{array}$ & $\begin{array}{l}\text { Factors } \\
\text { affecting dental } \\
\text { biofilm in } \\
\text { patients } \\
\text { wearing fixed } \\
\text { orthodontic } \\
\text { appliances }\end{array}$ & $\begin{array}{l}\text { Investigar a quantida- } \\
\text { de e a distribuição do } \\
\text { biofilme em pacientes } \\
\text { em uso aparelhos fixos } \\
\text { e sua relação com ida- } \\
\text { de, sexo, frequência de } \\
\text { escovação e motivação } \\
\text { do paciente. }\end{array}$ & $\begin{array}{l}\text { Estudo } \\
\text { transversal }\end{array}$ & $\begin{array}{l}52 \\
\text { pacientes } \\
\text { ortodônti- } \\
\cos \end{array}$ & $\begin{array}{l}\text { Pacientes que usam apa- } \\
\text { relhos ortodônticos fixos } \\
\text { têm o maior acúmulo de } \\
\text { biofilme principalmente na } \\
\text { área gengival e nas áreas } \\
\text { atrás dos fios do arco. }\end{array}$ & $\begin{array}{l}\text { O aparelho ortodôntico } \\
\text { é um dificultador da } \\
\text { higienização e favorece } \\
\text { o aumento do risco de } \\
\text { doenças bucais. }\end{array}$ \\
\hline $\begin{array}{l}\text { SHUKLA } \\
\text { et al., } 2017\end{array}$ & $\begin{array}{l}\text { Evaluation of } \\
\text { role of fixed } \\
\text { orthodontics in } \\
\text { changing oral } \\
\text { ecological flora } \\
\text { of opportunis- } \\
\text { tic microbes in } \\
\text { children and } \\
\text { adolescent }\end{array}$ & $\begin{array}{l}\text { Estimar a contagem e } \\
\text { o padrão de coloni- } \\
\text { zação de S. mutans } \\
\text { e Candida após a } \\
\text { aplicação de aparelhos } \\
\text { ortodônticos fixos e } \\
\text { comparar a eficácia } \\
\text { das escovas de dente } \\
\text { elétricas e manuais na } \\
\text { redução dos níveis de } \\
\text { microflora oral. }\end{array}$ & $\begin{array}{l}\text { Estudo } \\
\text { Caso-con- } \\
\text { trole }\end{array}$ & $\begin{array}{l}60 \\
\text { pacientes } \\
\text { ortodôn- } \\
\text { ticos, } 30 \\
\text { utilizando } \\
\text { escovas } \\
\text { manuais } \\
\text { e } 30 \\
\text { escovas } \\
\text { elétricas }\end{array}$ & $\begin{array}{l}\text { Após a instalação dos apa- } \\
\text { relhos ortodônticos fixos, } \\
\text { observa-se um aumento } \\
\text { significativo na coloniza- } \\
\text { ção fúngica e bacteriana. } \\
\text { O uso de escovas elétricas } \\
\text { favorece o controle dos } \\
\text { microorganismos. }\end{array}$ & $\begin{array}{l}\text { A escovação manual } \\
\text { é menos eficiente na } \\
\text { presença de aparelhos } \\
\text { ortodônticos, devendo } \\
\text { haver uma maior super- } \\
\text { visão dos pacientes. }\end{array}$ \\
\hline
\end{tabular}




\begin{tabular}{|c|c|c|c|c|c|c|}
\hline $\begin{array}{l}\text { SOLLEVELD } \\
\text { et al., } 2018\end{array}$ & $\begin{array}{l}\text { Are oral health } \\
\text { and fixed } \\
\text { orthodontic } \\
\text { appliances } \\
\text { associated with } \\
\text { sports injuries } \\
\text { and postural } \\
\text { stability in elite } \\
\text { junior male } \\
\text { soccer players? }\end{array}$ & $\begin{array}{l}\text { Examinar o possível } \\
\text { impacto de proble- } \\
\text { mas de saúde bucal e } \\
\text { uso de um aparelho } \\
\text { fixo ortodôntico em } \\
\text { lesões e estabilidade } \\
\text { postural do jogadores } \\
\text { de futebol. }\end{array}$ & $\begin{array}{l}\text { Estudo } \\
\text { transversal }\end{array}$ & $\begin{array}{l}187 \text { parti- } \\
\text { cipantes, } \\
\text { com idade } \\
\text { entre } 12 \\
\text { e } 17 \text { anos, } \\
\text { de clubes } \\
\text { de futebol } \\
\text { profissio- } \\
\text { nal belgas }\end{array}$ & $\begin{array}{l}\text { Foi encontrada associação } \\
\text { significativa entre lesões } \\
\text { ocorridas e estado de saúde } \\
\text { oral. Observou-se relação } \\
\text { indireta entre o uso de } \\
\text { aparelho fixo ortodôntico } \\
\text { e prejuízo na estabilidade } \\
\text { postural de atletas jovens. }\end{array}$ & $\begin{array}{l}\text { Problemas de saúde bu- } \\
\text { cal podem ser um fator } \\
\text { de risco para lesões e } \\
\text { que um aparelho fixo } \\
\text { ortodôntico tradicio- } \\
\text { nal pode dificultar o } \\
\text { desenvolvimento da } \\
\text { estabilidade postural } \\
\text { do corpo. }\end{array}$ \\
\hline $\begin{array}{c}\text { MADARIAGA } \\
\text { et al., } 2020\end{array}$ & $\begin{array}{l}\text { Impact of Fixed } \\
\text { Orthodontic } \\
\text { Appliance and } \\
\text { Clear Aligners } \\
\text { on the Perio- } \\
\text { dontal Health: } \\
\text { A Prospective } \\
\text { Clinical Study }\end{array}$ & $\begin{array}{l}\text { Avaliar a saúde perio- } \\
\text { dontal de pacientes or- } \\
\text { todônticos com terapia } \\
\text { periodontal de suporte } \\
\text { em um seguimento de } \\
3 \text { meses. }\end{array}$ & $\begin{array}{l}\text { Estudo } \\
\text { Caso-con- } \\
\text { trole }\end{array}$ & $\begin{array}{l}40 \text { pacien- } \\
\text { tes, } 20 \\
\text { utilizando } \\
\text { ortodontia } \\
\text { fixa e } 20 \\
\text { com ali- } \\
\text { nhadores } \\
\text { transpa- } \\
\text { rentes }\end{array}$ & $\begin{array}{l}\text { Os aparelhos fixos difi- } \\
\text { cultam a higienização dos } \\
\text { pacientes, devendo haver } \\
\text { uma rotina disciplinar su- } \\
\text { pervisionada de orientação } \\
\text { de higiene para manter um } \\
\text { controle de placa favorável. }\end{array}$ & $\begin{array}{l}\text { Pacientes ortodônticos } \\
\text { devem realizar uma } \\
\text { rotina de acompanha- } \\
\text { mento para garantir um } \\
\text { controle de higiene oral } \\
\text { adequado. }\end{array}$ \\
\hline
\end{tabular}

Fonte: construção do autor.

\section{RESULTADOS E DISCUSSÃO}

Este estudo buscou evidências sobre as vantagens e desvantagens da ortodontia fixa com braquetes e comparou com a técnica BOT, pensando em buscar alternativas de tratamento para o paciente atleta. Em alguns trabalhos, foi possível observar que, após a instalação dos dispositivos fixos, houve uma alteração quantitativa da flora bacteriana oral (TANNER et al., 2012; SHUKLA et al., 2017; MEI et al., 2017), além do aparecimento de lesões e inflamações bucais, peculiares à dificuldade de higienização impostas pelos aparatos ortodônticos (ZANATTA et al., 2014). Os resultados mostraram as vantagens e as desvantagens das técnicas vestibular e lingual dos dispositivos ortodônticos (Tabela 2), além de identificar a importância do uso do protetor bucal personalizado por indivíduos que utilizam aparelhos ortodônticos fixos, mesmo que sua modalidade esportiva não seja de combate, abrindo uma discussão para a busca de técnicas ortodônticas alternativas, com o intuito de minimizar fatores negativos, como o volume vestibular do protetor bucal sobre o aparelho tradicional. A figura 2 ilustra as características físicas dos diferentes tipos de aparelhos comparados no estudo. 
Tabela 2 - Comparativo entre as técnicas ortodônticas com e sem o uso de braquetes.

\begin{tabular}{|c|c|c|}
\hline Técnica & Vantagens & Desvantagens \\
\hline $\begin{array}{l}\text { Aparelho ortodôntico fixo com } \\
\text { braquetes por vestibular }\end{array}$ & $\begin{array}{l}\text { - Melhor controle de torque na retração } \\
\text { anterior; } \\
\text { - Facilidade de instalação dos acessórios; }\end{array}$ & $\begin{array}{l}\text { - Não favorece a estética; } \\
\text { - Dores nos lábios e bochechas; } \\
\text { - Dificuldade de utilização de fio dental e de higienização; } \\
\text { - Maior risco de gravidade durante um traumatismo; }\end{array}$ \\
\hline $\begin{array}{l}\text { Aparelho ortodôntico fixo com } \\
\text { braquetes por lingual }\end{array}$ & - Estética; & $\begin{array}{l}\text { - Maior tempo de procedimento; } \\
\text { - Desconforto do paciente; } \\
\text { - Procedimentos laboratoriais com custos elevados; } \\
\text { - Maior inclinação lingual dos incisivos durante a retração } \\
\text { anterior; } \\
\text { - Maior probabilidade de ferimentos na língua; } \\
\text { - Força maior durante a movimentação, preocupação } \\
\text { clínica; } \\
\text { - Interferência na fala; } \\
\text { - Reduz o espaço da língua; } \\
\text { - Dificuldade de utilização de fio dental e de higienização; }\end{array}$ \\
\hline $\begin{array}{l}\text { Bracketless Orthodontic Treat- } \\
\text { ment (BOT) }\end{array}$ & $\begin{array}{l}\text { - Maior conforto para o paciente; } \\
\text { - Estética preservada; } \\
\text { - Custo menor final; } \\
\text { - Redução do tempo de tratamento; } \\
\text { - Facilidade de higienização; } \\
\text { - Não interfere na fonação; }\end{array}$ & $\begin{array}{l}\text { - Maior tempo de consulta clínica (acima de } 30 \text { minutos); } \\
\text { - Necessidade de levante oclusal; }\end{array}$ \\
\hline
\end{tabular}

Fonte: construção do autor.

Figura 2 - Representação gráfica das três técnicas ortodônticas. A: ortodontia fixa por vestibular, indicando o volume ocupado pelo aparelho nesta região; B: ortodontia fixa lingual, mostrando a redução do espaço livre para o posicionamento da língua; C: técnica BOT, salientando a manutenção do espaço lingual e sem aumento do volume vestibular.

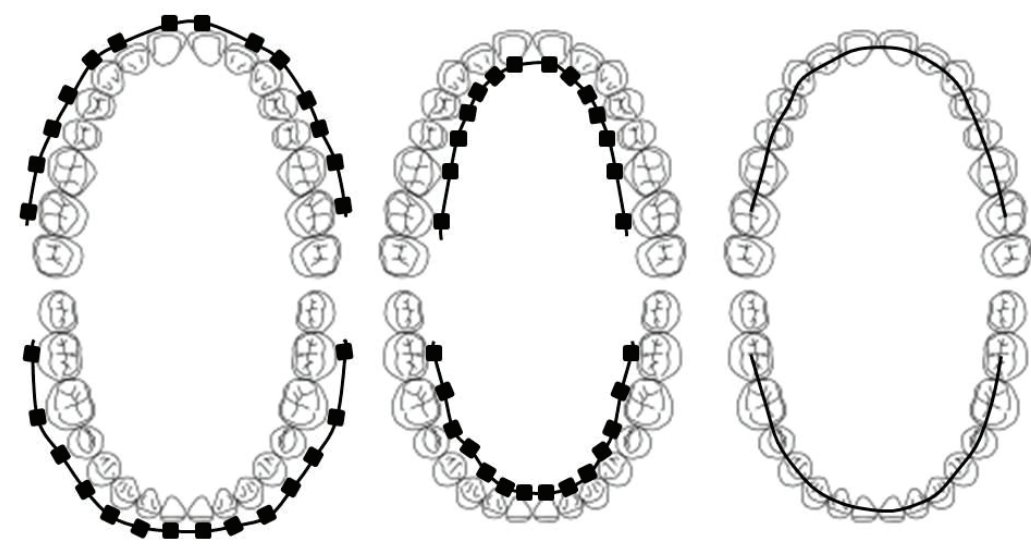

Fonte: Construção do autor.

A técnica vestibular de colagem ortodôntica, que imperou durante décadas, pela facilidade de instalação dos dispositivos e um melhor controle de torque durante a retração anterior, mostrou um 
aumento de acúmulo de biofilme, sobretudo em regiões críticas, como a localizada entre o braquete e a gengiva, o que a identifica como um fator de risco adicional importante, considerando a prevalência de inflamação gengival nesta região (MÁRTHA; MEZEI; JÁNOSI, 2013; ZANATTA et al., 2014). Os dados encontrados também mostraram um aumento das descalcificações dentárias com consequente aparecimento de lesões de manchas brancas (estágios iniciais de cáries), tendo como principal fator causador a dificuldade que a técnica ortodôntica impõe à higienização (AHMED; SAIF-UL-HAQUE; NAZIR, 2011; KHALAF et al., 2014; SALMERÓN-VALDÉS et al., 2016; ELTAYEB et al., 2017). Além disso, a dificuldade de utilizar o fio dental e de realizar uma escovação eficiente também foi responsável pela presença de halitose, problema que pode ter diferentes consequências, inclusive interferir no convívio social do esportista (ZURFLUH; WAES; FILIPPI, 2013; SÖKÜCÜ et al., 2016).

O aparelho fixo lingual é bastante utilizado com a intenção de preservar a estética. Mecanicamente, o método parece facilitar a correção de mordida profunda, da mordida cruzada e verticalização de molares (ALEXANDER et al., 1983). Além disso, as forças intrusivas aplicadas com a técnica lingual tendem a intruir os incisivos com menos inclinação vestibular do que na técnica tradicional (TANNE; LU; SAKUDA, 1992). Por outro lado, a maior proximidade do braquete lingual com o centro de resistência do dente pode ocasionar uma transmissão de força mais intensa ao ligamento periodontal, aumentando o risco de reabsorção radicular (PAIGE, 1982; RUMMEL; WIECHMANN; SACHDEVA, 1999). Considerando fatores como a manutenção da higiene oral e o risco de machucaduras associadas ao aparelho, não é possível observar muitas vantagens. A literatura indica que os aparelhos linguais podem causar uma maior dificuldade na fala e uma maior prevalência de lesões na língua. Outras questões pertinentes à dificuldade em comer, higiene bucal e o aparecimento de doenças com a cárie, são semelhantes aos aparelhos fixos vestibulares (LONG et al., 2013; MISTAKIDIS et al., 2016).

A dificuldade de higienização, percebida em pacientes que utilizam o aparelho com braquetes, pode levar ao aparecimento de gengivites e periodontites, aumentando o risco de problemas na saúde e no desempenho do atleta (GALLAGHER et al., 2018). Além disso, os longos períodos de treinamentos, onde também é comum o frequente consumo de repositores energéticos, contribui para a modificação do $\mathrm{pH}$ do meio bucal, favorecendo os processos de erosão química e aumentando o risco de doenças como a cárie dentária (ANTUNES et al., 2017; GALLAGHER et al., 2018). Dessa forma, a facilidade da higienização dos dentes deve ser preservada, da melhor maneira possível, e um aparelho ortodôntico com menos elementos pode ser mais favorável para a prevenção e manutenção da saúde bucal.

Tanto a técnica vestibular, como a lingual, expõem o indivíduo às lesões da mucosa oral oriundas de possíveis traumatismos ocorridos com a própria atividade esportiva realizada, podendo ocasionar prejuízos físicos e psicológicos (KVITTEN et al., 1998; SOLLEVELD et al., 2018). Além disso, existe a possibilidade de determinadas modalidades esportivas aumentarem os riscos de traumas orofaciais, ocasionando danos nos acessórios ortodônticos, expondo o atleta a um risco de inalação ou ingestão das peças ortodônticas e possibilitando, inclusive, que os dispositivos se alojem na região 
da orofaringe ou em outro local da cavidade oral, como já relatado em outras situações (CONTI et al., 2012; MCCARTHY; COBB, 2012; WILMOTT; IKEAGWUANI; MCLEOD, 2016). Assim, o profissional que irá atender o paciente atleta, que possui uma rotina onde é de fundamental importância o repouso entre os períodos de treinamentos e a concentração durante as competições, deve ter cuidado ao utilizar uma técnica ortodôntica que possa causar algum desconforto maior ou machucaduras adicionais no esportista.

Os braquetes ortodônticos favorecem o aumento da gravidade dos traumas ocorridos sobre os tecidos bucais (BARICEVIC et al., 2011), que são considerados frequentes durante a atividade esportiva. A etiologia dos traumas ocorridos na região orofacial tem sido o objetivo de muitos estudos, apresentando uma grande diversidade de fatores envolvidos. Os dados encontrados na literatura reforçam que a prática esportiva possui uma significativa influência sobre a quantidade e gravidade de lesões relatadas (BLINKHORN, 2000; GLENDOR, 2009), fazendo com que as medidas de proteção de lesões orofaciais, como o uso de protetores bucais personalizados, sejam indicadas (TANAKA; TSUGAWA; MAEDA, 2016). As técnicas adequadas de moldagem e confecção dos protetores bucais permitem que o dispositivo mantenha as suas propriedades de retenção e proteção contra lesões, preservando a comunicação e a respiração do esportista (KARAGANEVA et al., 2019). Contudo, quando um protetor bucal é confeccionado sobre um aparelho composto por braquetes, seu volume final fica maior inevitavelmente, o que pode ocasionar um desconforto para o atleta.

Buscando alternativas ortodônticas que eliminassem os braquetes, surge o aparelho fixo BOT trazendo inúmeras vantagens sobre as técnicas ortodônticas fixas como o conforto, a redução de lesões da mucosa oral e baixo custo, quando comparada às técnicas tradicionais. Trata-se de uma técnica ortodôntica ocluso-lingual onde o fio é fixado com resina fotopolimerizável, nas faces oclusais dos elementos posteriores e nas faces linguais dos anteriores, causando um pequeno levantamento oclusal (MUSILLI, 2008). Este levante por oclusal faz com que os contatos entre as cúspides dos dentes superiores e inferiores sejam liberados, facilitando os movimentos ortodônticos iniciais. O fato de não possuir acessórios ortodônticos que aumentem a retenção de placa bacteriana, facilita a higienização e constitui-se em uma excelente ferramenta para a movimentação dentária. Apesar de não utilizar braquetes, pode-se eventualmente instalar botões estéticos para auxiliar nos movimentos mecânicos com elásticos intermaxilares ou em forças binárias. Outro item importante nesta técnica inovadora é que seus acessórios não ocupam o espaço lingual, nem tampouco, possuem volumes por vestibular (JUNIOR et al., 2019).

Entretanto, uma desvantagem que deve ser considerada é o maior tempo de atendimento clínico para a instalação do aparelho. Este fator pode ocasionar desconforto ao paciente, podendo ser um empecilho para a mesma. Além disso, evidenciou-se que a colagem pela face lingual também pode dificultar a higienização no local, se o paciente não receber uma orientação de higiene adequada (MADARIAGA et al., 2020). 
O paciente atleta deve ser entendido de modo particular, pois a sua rotina de treinamento exige que este esteja em boas condições de saúde, para que possa direcionar, do melhor modo possível, os objetivos diante da melhoria do desempenho. O tratamento ortodôntico tem como objetivo a prevenção, a supervisão e a orientação do desenvolvimento do aparelho mastigatório, assim como a correção das estruturas dentofaciais, buscando a harmonia estética e a funcionalidade do complexo maxilo-mandibular. Todos estes elementos constituem fatores fundamentais que estão associados aos conceitos de saúde e qualidade de vida e, certamente, podem auxiliar o esportista no desenvolvimento de um melhor trabalho com maior segurança. Dessa forma, o aparelho fixo BOT, que é independe de braquetes, sugere ser uma promissora alternativa, para o tratamento ortodôntico destes indivíduos, em particular.

\section{CONSIDERAÇÕES FINAIS}

A odontologia conta com uma evolução e um crescente advento de materiais e tecnologias que visam oferecer cada vez mais tratamentos eficazes e rápidos para os pacientes. O sucesso de um tratamento odontológico deve considerar também o quanto este interferiu positiva ou negativamente sobre a vida ou a rotina do indivíduo, neste caso, o esportista.

Considerando a importância da ortodontia para o atleta profissional e amador e entendendo que a ortodontia convencional apresentou muitas desvantagens, podemos inferir que a técnica BOT pode ser uma interessante opção de tratamento ortodôntico, pois além de reduzir os riscos de injúrias às mucosas orais, permite uma boa higienização e possui um custo acessível, podendo ser indicada como uma nova opção de ortodontia do atleta.

\section{REFERÊNCIAS}

AHMED, I.; SAIF-UL-HAQUE; NAZIR, R. Carious lesions in patients undergoing orthodontic treatment. The Journal of the Pakistan Medical Association, v. 61, p. 1176-1179, 2011.

ALEXANDER, C. M.; ALEXANDER, R. G.; GORMAN, J. C.; HILGERS, J. J.; KURZ, C.; SCHOLZ, R. P.; SMITH, J. R. Lingual orthodontics: A status report. Part 5. Lingual mechanotherapy. Journal of clinical orthodontics, v. 17, n. 2, p. 99-115, 1983.

ANTUNES, L. S.; VEIGA, L.; NERY, V. S.; NERY, C. C.; ANTUNES, L. A. Sports drink consumption and dental erosion among amateur runners. Journal of Oral Science, v. 59, n. 4, p. 639-643, 2017. 
BARICEVIC, M.; STIPETIC, M. M.; MAJSTOROVIC, M.; BARANOVIC, M.; BARICEVIC, D.;

LONCAR, B. Oral mucosal lesions during orthodontic treatment. International Journal of Paediatric Dentistry, v. 21, p. 96-102, 2011.

BLINKHORN, F. A. The etiology of dento-alveolar injuries and factors influencing attendance for emergency care of adolescentes in the north west of England. Endodontics dental traumatology, v.16, p. 162-165, 2000.

CONTI, G.; DOLCI, M.; BORGONOVO, A.; MAIORANA, C. Aesthetic restoration of upper lip after removal of post-trauma foreign body (orthodontic bracket). European Journal of Paediatric Dentistry, v. 13, n. 3, p. 239-240, 2012.

ELTAYEB, M. K.; IBRAHIM, Y. E.; EL KARIM, I. A.; SANHOURI, N. M. Distribution of white spot lesions among orthodontic patients attending teaching institutes in Khartoum. BioMed Central Oral Health, v. 17, n. 1, p. 88, 2017.

GALLAGHER, J.; ASHLEY, P.; PETRIE, A.; NEEDLEMAN, I. Oral health and performance impacts in elite and professional athletes. Community Dentistry and Oral Epidemiology, v. 46, n. 6 , p. 563-568, 2018.

GLENDOR, U. Aetiology and risk factors related to traumatic dental injuries-a review of the literature. Dental Traumatology, v. 25, n. 1, p. 19-31, 2009.

JUNIOR, G. L. F.; TAVARES, N.; CAVALCANTE, G. R. G. C.; FONSECA, C. H. Técnica ortodôntica fixa sem braquetes, com tecnologia tridimensional "3DBOT". Orthodontics Science and Practice, v. 12 , n. 46 , p. 22-30, 2019.

KARAGANEVA, R.; PINNER, S.; TOMLINSON, D.; BURDEN, A.; TAYLOR, R.; YATES, J.; WINWOOD, K. Effect of mouthguard design on retention and potential issues arising with usability in sport. Dental Traumatology, v. 35, n. 1, p. 73-79, 2019.

KHALAF, K. Factors Affecting the Formation, Severity and Location of White Spot Lesions during Orthodontic Treatment with Fixed Appliances. Journal of Oral \& Maxillofacial Research, v. 5, n. 1, p. e4, 2014. 
KVITTEN, B.; HARDIE, N. A.; ROETTGER, M.; CONNY, J. Incidence of orofacial injuries in high school sports. Journal of Public Health Dentistry, v. 58, n. 4, p. 288-293, 1998.

LONG, H.; ZHOU, Y.; PYAKUREL, U.; LIAO, L.; JIAN, F.; XUE, J.; YE, N.; YANG, X.; WANG, Y.; LAI, W. Comparison of adverse effects between lingual and labial orthodontic treatment. Angle Orthodontic, v. 83, p.1066-1073, 2013.

MADARIAGA, A. C. P.; BUCCI, R.; RONGO, R.; SIMEON, V.; D’ANTÒ, V.; VALLETTA, R. Impact of Fixed Orthodontic Appliance and Clear Aligners on the Periodontal Health: A Prospective Clinical Study. Dental Journal (Basel), v. 8, n. 1, p. 4, 2020.

MÁRTHA, K.; MEZEI, T.; JÁNOSI, K. A histological analysis of gingival condition associated with orthodontic treatment. Romanian Journal of Morphology and Embryology, v. 54, n. 3 Suppl, p. 823-827, 2013.

MCCARTHY, E.; COBB, A. R. Orthodontic parotitis: a rare complication from an orthodontic appliance. Journal of Orthodontics, v. 39, n. 4, p. 314-316, 2012.

MEI, L.; CHIENG, J.; WONG, C.; BENIC, G.; FARELLA, M. Factors affecting dental biofilm in patients wearing fixed orthodontic appliances. Progress in Orthodontics, v. 18, n. 1, p. 4, 2017.

MISTAKIDIS, I.; KATIB, H.; VASILAKOS, G.; KLOUKOS, D.; GKANTIDIS, N. Clinical outcomes of lingual orthodontic treatment: a systematic review. European Journal of Orthodontics, v. 38, n. 5, p. 447-458, 2016.

MUSILLI, M. The Bracketless Fixed Orthodontics: nine years of clinical experimentation. Progress in Orthodontics, v. 9, n. 1, p. 72-91, 2008.

MUSILLI, M.; ACANFORA, M.; GHERLONE, E.; LUCCHESE, A, Anterior torque corretion with Bracketless fixed orthodontics. Journal of Clinical Orthodontics, v. 46, n. 6, p. 558-562, 2012.

PAIGE, S. F. A lingual light-wire technique. Journal of Clinical Orthodontics, v. 16, n. 8, p. 534 544, 1982. 
PAPAGEORGIOU, S. N.; GÖLZ, G.; JÄGER, U. M.; ELIADES, T.; BOURAUEL, C. Lingual vs. Labial Fixed Orthodontic Appliances: Systematic Review and Meta-Analysis of Treatment Effects. European Journal of Oral Sciences, v. 124, p. 105-118, 2016.

PARKER, K.; MARLOW, B.; PATEL, N.; GILL, D. S. A review of mouthguards: effectiveness, types, characteristics and indications for use. British Dental Journal, v. 222, n. 8, p. 629-633, 2017.

QUDEIMAT, M. A.; AlHASAN, A. A.; ALHASAN, M. A.; AL-KHAYAT, K.; ANDERSSON, L. Prevalence and severity of traumatic dental injuries among young amateur soccer players: A screening investigation. Dental Traumatology, v. 35, n. 4-5, p. 268-275, 2019.

RUMMEL, V.; WIECHMANN, D.; SACHDEVA, R. C. L. Precision finishing in lingual orthodontics. Journal of Clinical Orthodontics, v. 33, n. 2, p. 101-113,1999.

SALMERÓN-VALDÉS, E., LARA-CARRILLO, E., MEDINA-SOLÍS, C.; ROBLES-BERMEO, N. L.; SCOUGALL-VILCHIS, R. J.; CASANOVA-LOYOLA, A. P.; BARRERA, M. A. F. Tooth demineralization and associated factors in patients on fixed orthodontic treatment. Scientific Reports, v. 6, n. $36383,2016$.

SHALISH, M.; COOPER-KAZAZ, R.; IVGI, I.; CANETTI, L.; TSUR, B.; BACHAR, E.; CHAUSHU, S. Adult patients' adjustability to orthodontic appliances. Part I: a comparison between Labial, Lingual, and Invisalign ${ }^{\mathrm{TM}}$. European Journal of Orthodontics, v. 34, n. 6, p. 724-730, 2012.

SHEN, H.; SHAO, S.; ZHANG, J.; WANG, Z.; LV, D.; CHEN, W.; SVENSSON, P.; WANG, K. Fixed orthodontic appliances cause pain and disturbance in somatosensory function. European Journal of Oral Sciences, v. 124, n. 1, p. 26-32, 2016.

SHUKLA, C.; MAURYA, R.; SINGH, V.; TIJARE, M. Evaluation of role fixed orthodontics in changing oral ecological flora of opportunistic microbes in children and adolescente. Journal of Indian Society of Pedodontics and Preventive Dentistry, v. 35, n. 1, p. 34-40, 2017.

SÖKÜCÜ, O.; AKPINAR, A.; ÖZDEMIR, H.; BIRLIK, M.; ÇALIŞIR, M. The effect of fixed appliances on oral malodor from beginning of treatment till 1 year. BioMed Central Oral Health, v. 16, n. 14. 
SOLLEVELD, H.; FLUTTER, J.; GOEDHART, A.; VANDENBOSSCHE, L. Are oral health and fixed orthodontic appliances associated with sports injuries associated with sports injuries and postural stability in elite junior male soccer players? BioMed Central Sports Science, Medicine and Rehabilitation, v. 10, n. 16, 2018.

TANAKA, Y., TSUGAWA, T., MAEDA, Y. Effect of mouthguards on impact to the craniomandibular complex. Dental Traumatology, v. 33, n. 1, p. 51-56, 2016.

TANNE, K.; LU, Y. C. L.; SAKUDA, M. Biomechanical responses of tooth to orthodontic forces applied at the lingual bracket positions. Journal of Osaka University Dental School, v. 32, p. 6-13, 1992.

TANNER, A. C.; SONIS, A. L.; LIF HOLGERSON, P.; STARR, J. R.; NUNEZ, Y.; KRESSIRER, C. A.; PASTER, B. J.; JOHANSSON, I. White-spot lesions and gingivitis microbiotas in orthodontic patients. Journal of Dental Research, v. 91, n. 9, p. 853-858, 2012.

TIBANA, R. A.; PRESTES, J.; DE SOUSA, N. M. F.; DE SOUZA, V. C.; DE TOLÊDO NOBREGA, O.; BAFFI, M.; FERREIRA, C. E. S.; CUNHA, G. V.; NAVALTA, J. W.; TROMBETA, J. C. D. S.; CAVAGLIERI, C. R.; VOLTARELLI, F. A. Time-Course of Changes in Physiological, Psychological, and Performance Markers following a Functional-Fitness Competition. International Journal of Exercise Science, v. 12, n. 3, p. 904-918, 2019.

TOUGER-DECKER, R.; VAN LOVEREN, C. Sugars and dental caries. American Journal of Clinical Nutrition, v. 78, n. 4, p. 881S-892S, 2003.

VENABLES, M. C.; SHAW, L.; JEUKENDRUP, A. E.; ROEDIG-PENMAN, A.; FINKE, M.; NEWCOMBE, R. G.; PARRY, J.; SMITH, A. J. Erosive effect of a new sport drink on dental enamel during exercise. Medicine \& Science in Sports \& Exercise. v. 37, n. 1, p. 39-44, 2005.

WILMOTT, S. E.; IKEAGWUANI, O.; MCLEOD, N. M. H. An orthodontic bracket embedded in the medial pterygoid surface: a case report. European Journal of Orthodontics, v. 0, p. 1-3, 2015.

WU, A. K.; MCGRATH, C. P.; WONG, R. W.; RABIE, A. B.; WIECHMANN, D. A comparison of pain experienced by patients treated with labial and lingual orthodontic appliances. Annals of the Royal Australasian College of Dental Surgeons, v. 19, p. 76-178, 2008. 
ZANATTA, F. B.; ARDENGHI, T. M.; ANTONIAZZI, R. P.; PINTO, T. M. P.; ROSING, C. K. Association between gingivitis and anterior gingival enlargement in subjects undergoing fixed orthodontic treatment. Dental press journal of orthodontics, v. 19, n. 3, p. 59-66, 2014.

ZURFLUH, M.; WAES, H. J.M.; FILIPPI, A. The influence of fixed orthodontic appliances on halitosis. Schweizer Monatsschrift fur Zahnmedizin, v. 123, n. 12, p. 1064-1075, 2013. 
